\title{
Classification of Turkish Extra Virgin Olive Oils by a SAW Detector Electronic Nose
}

\author{
Pınar Kadiroğlu • Figen Korel · Figen Tokatlı
}

Received: 27 May 2010/Revised: 17 October 2010/Accepted: 19 October 2010/Published online: 5 November 2010 (C) AOCS 2010

\begin{abstract}
An electronic nose (e-nose), in combination with chemometrics, has been used to classify the cultivar, harvest year, and geographical origin of economically important Turkish extra virgin olive oils. The aroma fingerprints of the eight different olive oil samples [Memecik (M), Erkence (E), Gemlik (G), Ayvalık (A), Domat (D), Nizip (N), Gemlik-Edremit (GE), Ayvalık-Edremit (AE)] were obtained using an e-nose consisting a surface acoustic wave detector. Data were analyzed by principal component analysis (PCA) and discriminant function analysis (DFA). Classification of cultivars using PCA revealed that A class model was correctly discriminated from $\mathrm{N}$ in two harvest years. The DFA classified 100 and $97 \%$ of the samples correctly according to the cultivar in the 1st and 2nd harvest years, respectively. Successful separation among the harvest years and geographical origins were obtained. Sensory analyses were performed for determining the differences in the geographical origin of the olive oils and the preferences of the panelists. The panelists could not detect the differences among olive oils from two different regions. The cultivar, harvest year, and geographical origin of extra virgin olive oils could be discriminated successfully by the e-nose.
\end{abstract}

Keywords Extra virgin olive oil - Electronic nose . Classification - Principal component analysis . Discriminant function analysis - Sensory analysis

P. Kadiroğlu · F. Korel $(\bowtie) \cdot$ F. Tokatlı

Department of Food Engineering,

İzmir Institute of Technology, Urla, İzmir, Turkey

e-mail: figenkorel@iyte.edu.tr

\section{Introduction}

Recently, extra virgin olive oil (EVOO) has been in great demand by the consumers due to its nutritional, sensorial and functional properties. The desired aroma characteristics of EVOO are the result of the phenolic and volatile contents of the olive oil [1]. Factors such as cultivar, environment and cultural practices determine the quality and uniqueness of specific EVOOs [2]. The geographical origin of olive oil is one of the most significant factors affecting the aroma profile of olive oil [3].

The detection of the aromatic volatiles is important in EVOO quality control [1]. The conventional analytical methods that include GC [4], and GC/MS [5] and sensory analysis $[4,6,7]$ have been used for the geographical differentiation of EVOO. But these methods are often laborious and time consuming as well as requiring qualified staff. An e-nose providing fast, simple and easy sensory information in combination with chemometrics has been used for odor recognition and differentiation of EVOOs [8]. The discrimination among cultivars and geographical origin including adulteration, and authentication has mainly been proposed by Rezzi et al. [9]. Oliveros et al. [10] demonstrated the successful discrimination of different aromas of olive oils from five Mediterranean areas using an e-nose and chemometrics. Cosio et al. [2] characterized the geographical origin of Garda EVOOs by an e-nose and chemometrics. The discrimination capability of an e-nose using PCA has also been shown to analyze different Tuscan cultivars [7].

Sensory analysis has been defined as a scientific discipline which consists of a panel of trained or untrained panelists. Generally, it is used to discriminate olive oil with respect to its region of origin, variety, ripeness and extraction techniques [7]. The sensory quality of a food points to its desirability and acceptability. Color, taste and 
aroma are the main variables for the definition of the quality of olive oils [11]. Volatile compounds have a significant role in determination of correlation between the olive oil quality and sensory appreciation [7].

Several studies have been reported regarding the discrimination of olive oils produced in major olive oil producing countries such as Spain, Italy, and Greece using e-noses. Turkey is the fourth largest oil producing country (4.2\% olive oil production) and has provided $9.2 \%$ of the world export in the last decade [12]. Few studies have been published about olive oils produced in Turkey and none of these studies performed discrimination of olive oils using an e-nose. The objective of this study was to classify Turkish EVOOs according to olive cultivar, harvest year, and geographical origin using their aroma fingerprints obtained by an e-nose equipped with a surface acoustic wave (SAW) detector in combination with chemometrics. Sensory panels were conducted to determine any differences between the same cultivars of EVOOs obtained from different geographical origins. Consumers' preferences for the olive oils based on their color, odor, and taste attributes and their overall acceptability were also determined.

\section{Materials and Methods}

\section{Olive Cultivars}

The olive cultivars including Memecik (M), Erkence (E), Gemlik (G), Ayvalık (A), Domat (D), Nizip (N) were obtained from the Olive Research Institute (İzmir, Turkey). These cultivars except $\mathrm{N}$ are mainly cultivated in western part of Turkey. However, $\mathrm{N}$ is mainly cultivated in the southeastern part of Turkey, but $\mathrm{N}$ used in this study was obtained from an orchard in Izmir. Two olive cultivars, Ayvalık and Gemlik, were also obtained from the Olive Nursery in Edremit (located $\sim 160 \mathrm{~km}$ north of İzmir) and named as Ayvalık-Edremit (AE) and Gemlik-Edremit (GE) in order to determine the geographical differences among the same cultivars. About $15-25 \mathrm{~kg}$ olives of each cultivar were picked at once from olive trees at the same maturity level for two consecutive harvest years (2005/06 stated as 1st and 2006/07 stated as 2nd) and each cultivar was collected at the same time of the year. They were divided into $5 \mathrm{~kg}$ batches randomly for the extraction processes. The maturation level of each cultivar was determined by calculating the maturation index (MI, categories 0-7) according to Vinha et al. [13].

\section{Production of Olive Oil}

Olive oils were produced in a laboratory scale mill (maximum $5 \mathrm{~kg}$ capacity, TEM Spremoliva, Italy) in Food
Engineering Department of İzmir Institute of Technology. The olives were poured into the hopper and ground for $5 \mathrm{~min}$ at room temperature $\left(20-22^{\circ} \mathrm{C}\right)$. After grinding, the paste was stirred slowly for $45 \mathrm{~min}$ in the mill, where the microscopic oil drops joined together into bigger drops, which facilitated the mechanical extraction. The paste was then pressed by centrifugation and the water was separated from the oil during centrifugation and this process completed within $20 \mathrm{~min}$. At least two different batches of olive oils were obtained from each cultivar for each harvest year, stored in dark brown bottles at $8{ }^{\circ} \mathrm{C}$, and the headspaces of the bottles were flushed with nitrogen prior to analyses.

\section{Electronic Nose Analysis}

An e-nose $\left(z N o s e^{\text {TM }} 7100\right.$ vapor analysis system, EST, Newbury Park, CA, USA), consisting of a 1-m DB-5 column and a SAW detector with a parts per billion sensitivity, was used to obtain the aroma fingerprints of EVOO samples. Ten milliliters of each oil sample was transferred into a 40-ml septa-sealed screw cap vial and left overnight at room temperature prior to analysis. The vials were then placed in a water bath at $30{ }^{\circ} \mathrm{C}$ for $15 \mathrm{~min}$. During this time, the oil samples were allowed to equilibrate with the headspace in the vial and then the sample's vapor was pumped into the e-nose with a side-ported sampling needle $(5 \mathrm{~cm})$ through the septa. While the headspaces of the samples were equilibrated in the water bath, the system was calibrated with $n$-alkane calibration mixture (C6-C14) (SKA002190, EST, USA) before starting the sample measurements as well as in between each sample to ensure cleaning the system and a stable baseline. For each oil sample at least three vials were prepared and two readings were taken from each vial. After each run the detector was heated to $150{ }^{\circ} \mathrm{C}$ for $15 \mathrm{~s}$ for detector cleaning.

For each measurement, there were three phases: sampling, injection, and analysis. The sampling mode was set to $10 \mathrm{~s}$, after that the system was switched to a 10-s data acquisition mode and the inlet temperature was $200{ }^{\circ} \mathrm{C}$. While the sample was passing through the valve, the compounds were adsorbed onto the trap tube. Then the valve, set at $165^{\circ} \mathrm{C}$, was rotated to put the trap in line with the column for the injection phase. During the injection phase, the trap was heated to $280{ }^{\circ} \mathrm{C}$ in order to vaporize the adsorbed compounds. The compounds were transported to the capillary column (1 m, DB-5) with the carrier gas (purified helium) with a flow rate of $4.0 \mathrm{~cm}^{3} / \mathrm{min}$. The temperature of the column was programmed from 40 to $180{ }^{\circ} \mathrm{C}$ at a rate of $7{ }^{\circ} \mathrm{C} / \mathrm{s}$ and the compounds were separated and sequentially detected by the SAW detector $\left(20^{\circ} \mathrm{C}\right)$ through a deviation from its set frequency. The frequency profile read from the SAW detector was transformed to its first derivative. Each peak in the derivative plot corresponded to a specific volatile 
compound having a retention time specific for the column and analysis temperature. The area under the peak correlated with the concentration of the volatile compound, was expressed in counts [8].

Data were collected every $0.02 \mathrm{~s}$ using Microsense version 4 software (Newbury Park, CA, USA). The average of the duplicate e-nose measurements for each vial was calculated and a total of nine observations for each EVOO variety were used for the data analysis.

\section{Sensory Analysis}

Two different sensory analysis techniques were performed. First, the same-different discrimination test was used to determine whether there was any detectable difference between the EVOOs produced from the same cultivars grown in two different regions. The EVOOs used in this test were produced from Ayvalık and Gemlik olive cultivars which were grown in İzmir $(\mathrm{A}, \mathrm{G})$ and Edremit (AE, $\mathrm{GE}$ ) in two harvest years. The test was carried out with 30 untrained panelists who were chosen among the graduate students and faculty members (aged between 23 and 45) of the İzmir Institute of Technology. They were frequent olive oil consumers and agreed to taste olive oil by itself without a carrier such as bread or other food. With four EVOOs (A, $\mathrm{AE}$ and $\mathrm{G}, \mathrm{GE}$ ), six combinations of different sets were possible. Two pairs of EVOO samples were presented to the panelists in glasses at each session and one of the pairs contained identical samples (A-A, AE-AE, G-G or GE$\mathrm{GE}$ ), and the second pair contained the samples that differ in the growing region (A-AE or $\mathrm{G}-\mathrm{GE}$ ). A total of 60 pairs was presented to the panelists and the pairs having identical and different pairs were randomly selected. The panelists were asked to evaluate the samples and determine whether they were the same or different samples by examining their color, odor and flavor. Re-tasting was allowed and they were instructed to rinse their mouths with tap water (at room temperature) and to neutralize their palates between samples by eating unsalted bread. Two sessions were conducted for each harvest year and the second session was conducted $15 \mathrm{~min}$ after completion of the first session.

Next, the acceptance test was conducted to establish the panelists' preference between eight different EVOO samples (M, E, G, A, D, N, GE, and AE) with respect to their color, odor, taste and overall acceptability. A total of 20 panelists was selected among the ones who had attended the same-different discrimination test and had discriminated the samples successfully. EVOO samples were presented and they were asked to define their preferences based on color, odor, taste, and overall acceptance according to the categorical scale ranging from excellent (1) to very bad (5). Panelists were instructed to rinse their mouths with tap water (at room temperature) and to neutralize their palates between samples by eating unsalted bread. Two sessions were performed for each harvest year on two consecutive days and in each session a total of four samples were allowed to be tested. The samples were stored at $8{ }^{\circ} \mathrm{C}$, and the headspaces of the bottles were flushed with nitrogen prior to the sessions.

\section{Data Analysis}

PCA and DFA were performed to discriminate EVOO samples based on their aroma fingerprints obtained by the e-nose using SIMCA software (Umetrics, Umeå, Sweden) and Statistica software (StatSoft Inc., Tulsa, OK, USA), respectively. PCA is a multivariate projection method reducing the number of variable dimensionality to a smaller number of principal components (PCs) by effective visualization, regression and classification of multivariate data [14]. Soft independent modeling of class analogy (SIMCA) is used as a class-modeling tool. SIMCA calculates the PCs for each category separately and model functions for each class are calculated using a specified number of PCs. Coomans' plot is a graphical display constructed using the PCA class models used for the classification of cultivars, harvest years or geographical origins. The significant PCs of each category build the class model after a separate scaling of each category. In Coomans' plot, class distances for two classes (class 1 and class 2) are plotted against each other in a scatter plot and both axes on the plot indicate the critical distances [15].

DFA is a supervised classification technique, where the number of categories and samples that belong to each category are previously defined. The criterion of DFA for selection of variables is a maximum differentiation between the categories and minimizes the variance within the categories. A number of orthogonal discriminant functions (DFs), equal to the number of categories minus one, are obtained and this allows the samples to be classified in one or another category. Two or three DFs are used to map the data in two or three dimensional plots and observe separation between categories. Correct classification rates are calculated [16, 17].

The data of the same-different discrimination test and acceptance test were analyzed using the Chi-square test and ANOVA using MINITAB ${ }^{\circledR}$ release 14 (Minitab Inc., State College, PA, USA), respectively.

\section{Results and Discussion}

\section{Electronic Nose Analysis}

The aroma fingerprints of eight different oils were obtained by an e-nose equipped with a SAW detector. Figure 1 


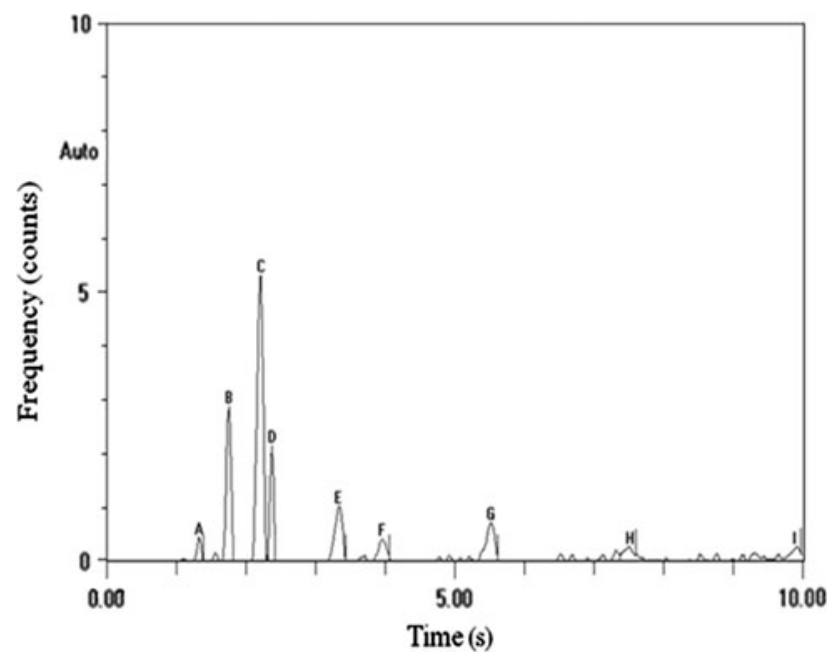

Fig. 1 A typical e-nose chromatogram of EVOO
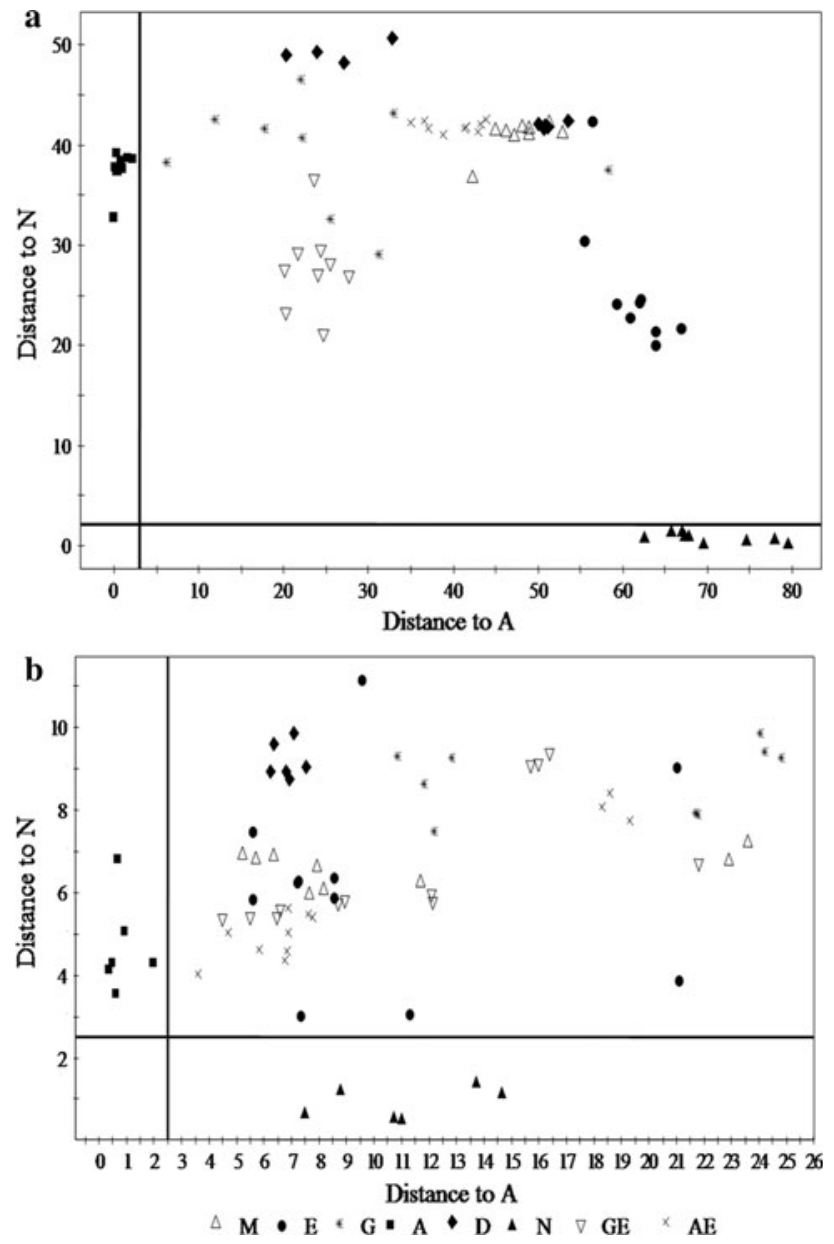

Fig. 2 Coomans' plot for the classification of $\mathrm{N}$ and A olive oils of the 1st harvest year (a) and the 2nd harvest year (b). [(a) PCA of A: $p=7, r_{\mathrm{x}}^{2}=0.998$ and $\mathrm{N}: p=4, r_{\mathrm{x}}^{2}=0.976$; (b) PCA of A: $p=3$, $r_{\mathrm{x}}^{2}=0.958$ and $\left.\mathrm{N}: p=3, r_{\mathrm{x}}^{2}=0.927\right]$
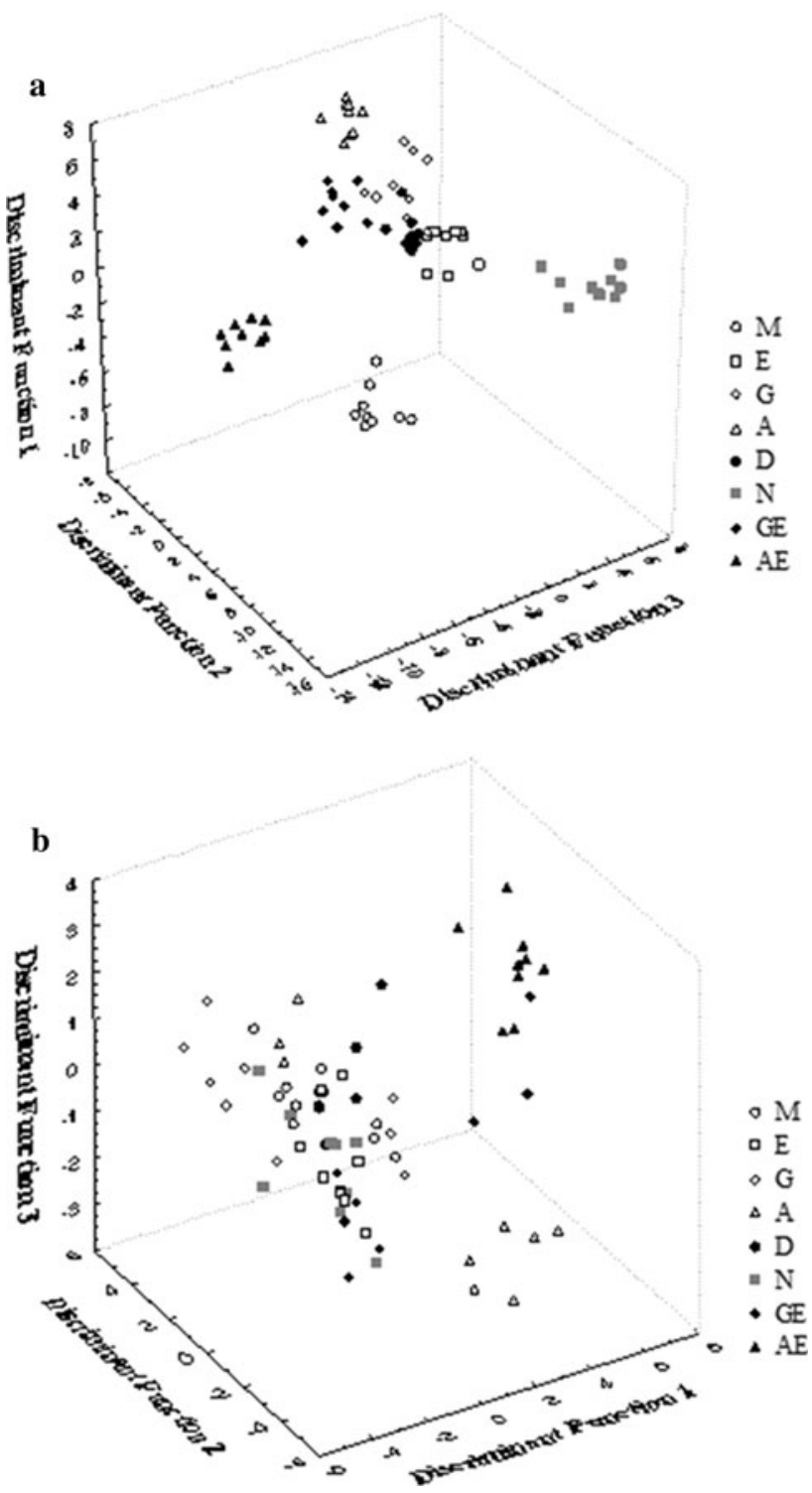

Fig. 3 3-D DFA of EVOOs based on cultivar produced in the 1st harvest year (a) and 2nd harvest year (b) [Plot of the first three discriminant functions (DFs).]

shows the typical chromatogram of an EVOO sample. Total of 15 peaks in all the chromatograms were considered and their areas were counted. Among them, six were common compounds for all the samples and the rest were seen in some oil samples. In fact, different EVOOs showed variations in the amount of every compound.

The aroma fingerprints of eight different EVOO samples were analyzed using PCA to see the discrimination of the samples based on the cultivar. Using the PCA class model, the Coomans' plot was constructed for the classification of $\mathrm{A}$ and $\mathrm{N}$ olive oils in two consecutive harvest years. Model parameters and Coomans' plots are shown in Fig. 2. This 
Fig. 4 Coomans' plot for the classification of EVOOs of the 1 st and 2nd harvest year. [PCA of 1st harvest year: $p=5$, $r_{\mathrm{x}}^{2}=0.825$ and 2 nd harvest year: $\left.p=4, r_{\mathrm{x}}^{2}=0.731\right]$

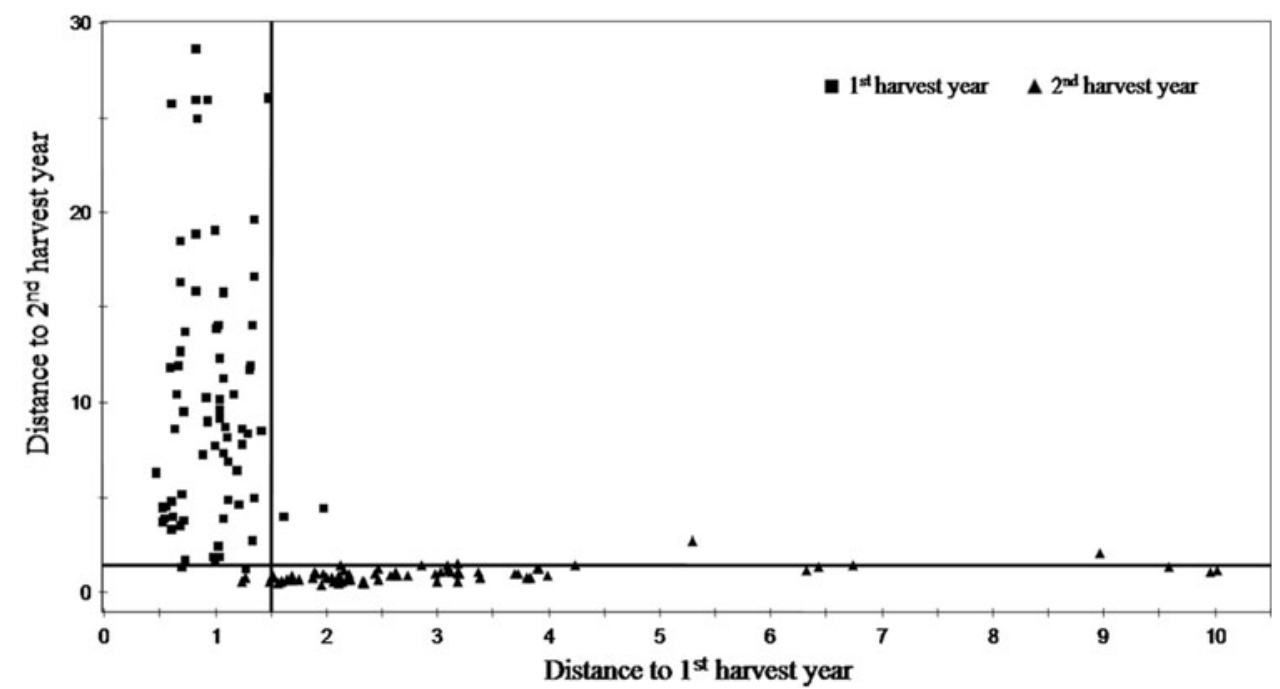

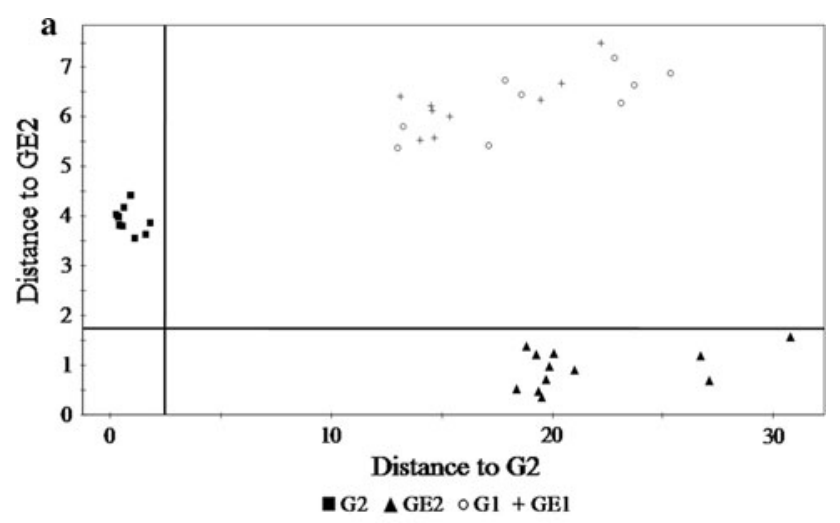

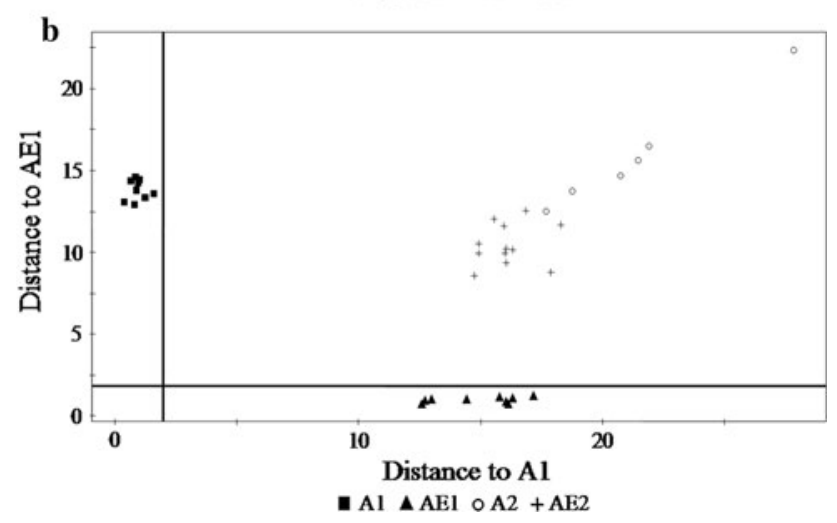

Fig. 5 Coomans' plot for the classification of EVOOs according to geographical origin and harvest year for $\mathrm{G}(\mathbf{a})$ and $\mathrm{A}(\mathbf{b})$ olive oils. [(a) PCA of G2: $p=5, r_{\mathrm{x}}^{2}=0.993$ and GE2: $p=2, r_{\mathrm{x}}^{2}=0.904$; (b) PCA of A1: $p=3, r_{\mathrm{x}}^{2}=0.861$ and AE1: $\left.p=2, r_{\mathrm{x}}^{2}=0.810\right]$

figure reveals that the A class model was correctly discriminated from the $\mathrm{N}$ in two harvest years. Additionally, a similar discrimination pattern was visualized among the other olive oil samples obtained from different cultivars. The clear separation among $\mathrm{A}$ and $\mathrm{N}$ models can be attributed to the difference in the cultivation areas of these oil samples. A is the most commonly consumed cultivar in the north-west region and $\mathrm{N}$ is mainly cultivated in the south-east region of Turkey and is the least commonly consumed cultivar. A previous study on discrimination of Tuscan single-cultivar EVOOs using an e-nose equipped with five different micro-sensors revealed that classification of these cultivars was possible [7].

Using DFA, the aroma fingerprints of eight different EVOO samples were also analyzed to see the discrimination of the samples based on the cultivar. As observed in Fig. 3, the DFA results represented as a 3-D plot of DFs allowed us to obtain well-defined and separated clusters for each olive cultivar. In the 1st harvest year, the cultivars were grouped with a $100 \%$ correct classification rate (Fig. 3a). However, in the 2nd harvest year, the correct classification rate was 97\%. One of the samples of M (Memecik) and N (Nizip) cultivars were grouped in E (Erkence) and GE (GemlikEdremit), respectively (Fig. 3b).

The comparison of the EVOO samples in two harvest years was obtained by applying Coomans' plot (Fig. 4) to the e-nose data. The $x$-axis of the Coomans' plot corresponded to the distance to the 1st harvest year, the $y$-axis showed the distance to the 2nd harvest year. The model parameters are given in Fig. 4. The EVOO samples of 2nd harvest year were grouped in the lower-right hand side of the plot. The EVOO samples of 1st harvest year were clustered in the upper-left hand side of the plot. This figure shows that the harvest year is effective on the discrimination of the EVOOs. It is well known that the changes in the climate during each year affect the aroma profile of the olive oils [18].

The model parameters and Coomans' plot of the $\mathrm{G}$ and A olive oil samples obtained from the same olive cultivars collected from two different orchards located in İzmir and Edremit regions in two consecutive harvest years are presented in Fig. 5. G and A olive oils obtained from İzmir 
Table 1 Sensory scores for the EVOOs of the 1st and 2nd harvest years
Data $=$ Means \pm SD

$M$ memecik, $E$ erkence, $G$ gemlik, $A$ ayvalık, $D$ domat, $N$ nizip, $G E$ gemlik-edremit, $A E$ ayvalık-edremit

Column means having a different letter or letters differ significantly $(p<0.05)$

A Sensory scores range from 1 (excellent) to 5 (very bad)

\begin{tabular}{|c|c|c|c|c|}
\hline \multirow[t]{2}{*}{ Olive oil samples } & \multicolumn{4}{|l|}{ Sensory scores ${ }^{\mathrm{A}}$} \\
\hline & Color & Odor & Taste & Overall acceptance \\
\hline \multicolumn{5}{|l|}{ 1st harvest year } \\
\hline $\mathrm{M}$ & $2.05 \pm 0.60^{\mathrm{a}}$ & $2.45 \pm 0.76^{\mathrm{a}}$ & $2.80 \pm 1.01^{\mathrm{a}}$ & $2.68 \pm 0.67^{\mathrm{ab}}$ \\
\hline $\mathrm{E}$ & $2.00 \pm 1.08^{\mathrm{a}}$ & $2.45 \pm 0.69^{\mathrm{a}}$ & $2.95 \pm 1.19^{\mathrm{a}}$ & $2.84 \pm 1.01^{\mathrm{bc}}$ \\
\hline $\mathrm{G}$ & $2.30 \pm 0.80^{\mathrm{a}}$ & $2.60 \pm 0.94^{\mathrm{a}}$ & $3.00 \pm 0.97^{\mathrm{a}}$ & $2.89 \pm 0.81^{\mathrm{bc}}$ \\
\hline A & $2.15 \pm 0.75^{\mathrm{a}}$ & $2.50 \pm 1.00^{\mathrm{a}}$ & $3.25 \pm 1.21^{\mathrm{a}}$ & $2.95 \pm 0.94^{\mathrm{b}}$ \\
\hline $\mathrm{D}$ & $2.30 \pm 0.57^{\mathrm{a}}$ & $2.30 \pm 0.73^{\mathrm{a}}$ & $2.55 \pm 0.76^{\mathrm{a}}$ & $2.40 \pm 0.68^{\mathrm{ac}}$ \\
\hline $\mathrm{N}$ & $2.50 \pm 0.83^{\mathrm{a}}$ & $3.20 \pm 1.32^{\mathrm{b}}$ & $2.80 \pm 1.47^{\mathrm{a}}$ & $3.05 \pm 1.31^{\mathrm{b}}$ \\
\hline GE & $2.05 \pm 0.60^{\mathrm{a}}$ & $2.60 \pm 0.95^{\mathrm{a}}$ & $2.40 \pm 0.82^{\mathrm{a}}$ & $2.33 \pm 0.69^{\mathrm{a}}$ \\
\hline $\mathrm{AE}$ & $2.15 \pm 0.67^{\mathrm{a}}$ & $2.25 \pm 0.72^{\mathrm{a}}$ & $2.50 \pm 0.89^{\mathrm{a}}$ & $2.35 \pm 0.61^{\mathrm{ac}}$ \\
\hline \multicolumn{5}{|l|}{ 2nd harvest year } \\
\hline M & $2.10 \pm 0.45^{\mathrm{ab}}$ & $2.40 \pm 0.82^{\mathrm{a}}$ & $2.50 \pm 0.76^{\mathrm{a}}$ & $2.47 \pm 0.77^{\mathrm{ab}}$ \\
\hline E & $1.85 \pm 0.87^{\mathrm{a}}$ & $2.50 \pm 1.19^{\mathrm{a}}$ & $3.45 \pm 1.14^{\mathrm{b}}$ & $3.10 \pm 1.15^{\mathrm{b}}$ \\
\hline G & $2.15 \pm 0.67^{\mathrm{ab}}$ & $2.20 \pm 0.69^{\mathrm{a}}$ & $2.40 \pm 0.82^{\mathrm{a}}$ & $2.37 \pm 0.68^{\mathrm{a}}$ \\
\hline A & $2.20 \pm 0.52^{\mathrm{ab}}$ & $2.50 \pm 0.68^{\mathrm{a}}$ & $2.30 \pm 0.57^{\mathrm{a}}$ & $2.25 \pm 0.44^{\mathrm{a}}$ \\
\hline $\mathrm{D}$ & $3.15 \pm 0.81^{\mathrm{c}}$ & $2.65 \pm 0.93^{\mathrm{a}}$ & $2.75 \pm 0.72^{\mathrm{a}}$ & $2.79 \pm 0.63^{\mathrm{ab}}$ \\
\hline $\mathrm{N}$ & $1.90 \pm 0.78^{\mathrm{a}}$ & $2.74 \pm 0.87^{\mathrm{a}}$ & $2.95 \pm 1.09^{\mathrm{a}}$ & $2.68 \pm 1.06^{\mathrm{ab}}$ \\
\hline GE & $2.05 \pm 0.39^{\mathrm{ab}}$ & $2.40 \pm 0.82^{\mathrm{a}}$ & $2.60 \pm 0.99^{\mathrm{a}}$ & $2.53 \pm 0.90^{\mathrm{ab}}$ \\
\hline $\mathrm{AE}$ & $2.40 \pm 0.94^{\mathrm{ab}}$ & $2.40 \pm 0.94^{\mathrm{a}}$ & $2.55 \pm 0.76^{\mathrm{a}}$ & $2.53 \pm 0.69^{\mathrm{ab}}$ \\
\hline
\end{tabular}

and Edremit were able to be separated clearly. This result demonstrated the effect of geographical origin on the same cultivar of olive oil. Figure 5 also suggests that these olive oil samples were able to be separated with respect to the harvest year since $\mathrm{G}$ and GE olive oils harvested in the 1st year were clustered beyond the critical limits in Fig. 5a. Similarly A and AE olive oils harvested in the 2nd year were plotted beyond the critical limits (Fig. 5b). In previous studies, the successful discrimination ability of an e-nose associated with chemometrics used for classification of the olive oil samples from different cultivars and geographical origins were also reported [19, 20].

\section{Sensory Analysis}

The panelists' scores of the same-different test for the A and $\mathrm{G}$ olive oil samples of the 1 st and 2 nd harvest years were evaluated by the Chi-square test. Based on the panelists' scores, the Chi-square test results for $\mathrm{A}$ and $\mathrm{AE}$ olive oils were 0.07 and 0.27 for the 1 st and 2 nd harvest years, respectively. The Chi-square test results for $\mathrm{G}$ and $\mathrm{GE}$ oils were 0.31 for the 1 st harvest year and 1.07 for the 2nd harvest year. The associated Chi-square value was 3.84 ( $d f=1, \alpha=0.05$ ) and the results were lower than this value [21]. The results showed that there were no differences distinguished between the EVOO samples obtained from $A$ and $G$ olives cultivated in two different geographical regions (İzmir and Edremit) based on the panelists' responses. It could be concluded that no differences were observed related to the effect of environmental and location conditions on the sensorial quality of these olive oils by the panelists.

Based on the acceptance test results, there were no significant differences among the samples based on their color, odor and taste attributes in the 1st harvest year. In addition, the difference between the odors of the samples was not recognized by the panelists in the 2nd harvest year (Table 1). Only N showed a different odor property among the olive oil samples. When the overall acceptance results were compared, significant differences were observed among the samples $(p<0.05)$. E olive oil samples were preferred by the panelists for their color. AE and $G$ olive oil samples were preferred for their odor in the 1st and 2nd harvest year, respectively. GE olive oil sample in the 1st harvest year and A olive oil in the 2nd harvest year were mostly liked for their taste and also the most preferred among olive oils. Caporale et al. [6] worked with a panel of consumers familiar with several typical EVOOs to assess the impact of information about the origin of the product on the sensory profile perception and it was shown that the origin affected the expectations based on the specific sensory attributes in consumers familiar with EVOO.

\section{Conclusion}

The results revealed that EVOOs obtained from different cultivars were able to be classified by PCA class model as well as with $97-100 \%$ correct classification rate between different cultivars by DFA. G and A EVOO samples 
obtained from two different regions (İzmir and Edremit) were also discriminated successfully. Sensory evaluation of these olive oil samples showed that the effect of geographical origin on the sensorial properties of olive oil samples could not be distinguished by the panelists. The acceptance test results showed that G/GE and A/AE olive oil samples obtained either from İzmir or Edremit were liked most by the panelists in the 1st and 2 nd harvest years. As a conclusion, the e-nose in combination with chemometrics could be used to classify Turkish EVOO samples based on their aroma fingerprints with respect to the olive cultivar, geographical origin and harvest year. Although in order to validate fully the usefulness of an e-nose for the classification of olive oils, larger sets of data should be processed to improve the precision of the classification models developed in this study.

Acknowledgments This research was supported by the Scientific and Technical Research Council of Turkey (TÜBITAK, Project No. 1040333). The authors would like to thank the Olive Research Institute (Izmir) and the Olive Nursery (Edremit) for providing the olive samples. The authors would also like to thank the sensory panel members for their participation.

\section{References}

1. Morales MT, Rios JJ, Aparicio RJ (1997) Changes in the volatile composition of virgin olive oil during oxidation: flavors and offflavors. J Agric Food Chem 45:2666-2673

2. Cosio MS, Ballabio D, Benedetti S, Gigliotti C (2006) Geographical origin and authentication of extra virgin olive oils by an electronic nose in combination with artificial neural networks. Anal Chim Acta 567:202-210

3. Araghipour N, Colineau J, Koot A, Akkermans W, Rojas JMM, Beauchamp J, Wisthaler A, Märk TD, Downey G, Guillou C, Mannina L, van Ruth S (2008) Geographical origin classification of olive oils by PTR-MS. Food Chem 108:374-383

4. Angerosa F, Mostallino RC, Basti RV (2000) Virgin olive oil odour notes: their relationships with volatile compounds from the lipoxygenase pathway and secoiridoid compounds. Food Chem 68:283-287

5. Tura D, Prenzler PD, Bedgood DR Jr, Antolovich M, Robards K (2004) Varietal and processing effects on the volatile profile of Australian olive oils. Food Chem 84:341-349

6. Caporale G, Policastro S, Carlucci A, Monteleone E (2006) Consumer expectations for sensory properties in virgin olive oils. Food Qual Pref 17:116-125

7. Cimato A, Dello Monacoa D, Distante C, Epifani M, Siciliano P, Taurino AM, Zuppa M, Sani G (2006) Analysis of single-cultivar extra virgin olive oil by means of an electronic nose and HS-SPME/GC/MS methods. Sens. Actuators B 114:674-680

8. Gan HL, Che Man YB, Tan CP, NorAini I, Nazimah SAH (2005) Characterisation of vegetable oils by surface acoustic wave sensing electronic nose. Food Chem 89:507-518

9. Rezzi S, Axelson DE, Héberger K, Reniero F, Mariani C, Guillou C (2005) Classification of olive oils using high throughput flow ${ }^{1} \mathrm{H}$ NMR fingerprinting with principal component analysis, linear discriminant analysis and probabilistic neural networks. Anal Chim Acta 552:13-24

10. Oliveros CC, Boggia R, Casale M, Armanino C, Forina M (2005) Optimization of a new headspace mass spectrometry instrument discrimination of different geographical origin olive oils. J Chromatogr A 1076:7-15

11. Angerosa F (2000) Sensory quality of olive oil. In: Harwood J, Aparicio R (eds) Handbook of olive oil: analysis and properties. Aspen Publishers, Gaithersburg, pp 355-356

12. International Olive Council (2010). http://www.international oliveoil.org

13. Vinha AF, Ferreres F, Silva BM, Valentao P, Gonçalves A, Pereria JA, Oliveira MB, Seabra RM, Andrade PB (2005) Phenolic profiles of Portuguese olive fruits (Olea europaea L.): influences of cultivar and geographical origin. Food Chem 89:561-568

14. Poulli KI, Mousdis GA, Georgiou CA (2005) Classification of edible and lampante virgin olive oil based on synchronous fluorescence and total luminescence spectroscopy. Anal Chim Acta 542:151-156

15. Eriksson L, Johansson E, Kettaneh-Wold N, Wold S (2001) Multi- and mega-variate data analysis: principles and applications. Umetrics Academy, Umeå, p 533

16. Meloun M, Militký J, Forina M (1992) Exploratory and factor analysis of multivariate data. In: chemometrics for analytical chemistry, Vol 1: PC-aided statistical data. Ellis Horwood, Chichester, England, pp 212-300

17. Gardner JW, Hines EL (1997) Pattern analysis techniques. In: Kress-Rogers E (ed) Handbook of biosensors and electronic noses, medicine, food, and the environment. CRC Press, Boca Raton, pp 633-652

18. Tura D, Failla O, Bassi D, Pedó S, Serraiocco A (2009) Environmental and seasonal influence on virgin olive (Olea europaea L.) oil volatiles in northern Italy. Sci Hortic 122:385-392

19. García-González DL, Aparicio R (2004) Classification of different quality virgin olive oils by metal-oxide sensors. Eur Food Res Technol 218:484-487

20. Casale M, Armanino C, Casolino C, Forina M (2007) Combining information from headspace mass spectrometry and visible spectroscopy in the classification of the Ligurian olive oils. Anal Chim Acta 589:89-95

21. Meilgaard M, Civille GV, Carr BT (1999) Overall difference tests VI. Same/different test (or simple difference test). In: Sensory evaluation techniques, 3rd edn. CRC Press, NY, pp 79-82 